\title{
DNA polymerase 5 acetylation by Eso1 is essential for Schizosaccharomyces pombe viability
}

\author{
ZHIMING CHEN ${ }^{1}$, HONGSHI CAO ${ }^{2}$, YINGQIANG LU ${ }^{1}$, QIANG REN ${ }^{1}$ and LIANKUN SUN ${ }^{1}$ \\ ${ }^{1}$ Department of Forensic Pathology, Basic Medical College, and ${ }^{2}$ Department of Neurosurgery, \\ The First Hospital, Jilin University, Jilin 130021, P.R. China
}

Received October 23, 2015; Accepted July 24, 2017

DOI: $10.3892 /$ ijmm.2017.3192

\begin{abstract}
Eco1/Eso1 protein plays an important role in chromosome segregation, DNA repair and gene regulation. Ecol mutation induces Roberts syndrome clinically and rDNA transcription disorders in vivo. In this study, we examined the role of Esol protein binding to polymerase 5 (Pol5) and the acetylation of Pol5 protein in the regulation of Schizosaccharomyces pombe (S. pombe) viability. Immunoprecipitation and mass spectrometry assays identified Esol protein binding to $\mathrm{Cdc} 2$, Pol5 and Cdc21, as well as other proteins. Pol5 protein specifically bound to Eso1 protein, but not to the Rad30 part or Rad30 part plus the additional zinc finger domain of Ecol protein. Mass spectrometry data further identified several acetylation or trimethylation modification sites in the lysine residues of the Pol5 protein. However, the mutation of the Pol5 K47 site to arginine was lethal to $S$. pombe. Esol protein was able to acetylate Pol5 protein and mediate $S$. pombe viability. On the whole, our data indicate that the Esol interaction with Pol5 which acetylates Pol5 protein is essential for $S$. pombe viability.
\end{abstract}

\section{Introduction}

Eco1/Eso1 protein plays an important role in chromosome segregation, DNA repair and gene regulation (1-3). Eco1/Eso1 protein mediates the formation of the cohesion complex during DNA replication, chromosome separation and cell mitosis from the metaphase to the anaphase transition of the cell cycle. The cohesion complex is critical for the faithful chromosome segregation between two sister chromatids at the anaphase (4), consists of 4 highly conserved proteins (Smc3, Smc1, Scc3 and Scc1) $(5,6)$, and functions to entrap the chromatids by forming a topological ring (7) during the cell cycle. Eco1/ Esol protein is an acetyltransferase and is able to acetylate

Correspondence to: Dr Zhiming Chen or Dr Liankun Sun, Department of Forensic Pathology, Basic Medical College, Jilin University, Xinjiang Street 126, Changchun, Jilin 130021, P.R. China E-mail: chenzm@jlu.edu.cn

E-mail:sunlk@jlu.edu.cn

Key words: Eso1, polymerase 5, acetylation, rDNA transcription, tandem affinity purification, Schizosaccharomyces pombe the two conserved lysine sites in the Smc3 protein, enabling Smc3 to form the cohesion complex (8-11). A previous study revealed that mutations of $E S C O 2$, the human ortholog of Eco1, were associated with a human developmental disorder known as Roberts syndrome (12). The role of Eco1/Eso1 in the contribution to chromosome structure and organization was then proposed (13). For example, our previous genetics screening data also demonstrated an intimate association between cohesion and chromatin separation (14). Recently, rRNA transcription and protein translation defects occurred in budding yeast that carry Roberts syndrome-related Ecol mutations and in cells from patients with Roberts syndrome $(15,16)$. These data indicate that Eco1/Esol protein may play an important role in Schizosaccharomyces pombe (S. pombe) viability. However, the molecular mechanisms through which Eco1 protein functions in $S$. pombe, remain to be defined. For example, determining how and which protein Ecol/Esol protein binds to or interacts with, and any additional functions of Ecol/Esol protein may shed light into this matter.

In the present study, we first purified Esol protein using a tandem affinity purification (TAP) tag antibody and immunoprecipitated whole cell extracts from the wild-type $S$. pombe strain to identify Esol binding partners. We then focused on one of its interacting proteins, polymerase 5 (Pol5), as the Pol5 protein provides a conserved function in rDNA transcription in yeast $(17,18)$. In addition, Myb-binding protein 1a (Mybbpla), a human homologue of Pol5, has been shown to function in rDNA transcription and processing, and to be associated with early embryonic development and carcinogenesis $(19,20)$. Subsequently, we assessed how Esol interacts with Pol5 and and examined alterations in Pol5 protein acetylation, and the effects of these on $S$. pombe viability. This study aimed to provide insightful information regarding the interaction of Esol with Pol5 and the acetylation of Pol5 protein, and the effects of these on $S$. pombe viability. Future studies are also required in order to investigate whether and how Esol acetylates the Pol5 K47 site for S. pombe survival.

\section{Materials and methods}

Strains and culture media. The $S$. pombe strains used in this study are listed in Table I and were cultured in standard conditions, as previously described (21). For $S$. pombe transformation, the lithium acetate protocol was utilized, as described 
in a previous study (22). pDblet (acquired from Baumann's Lab, Stowers Institute, Kansas City, MO, USA) was used as a plasmid vector for the expression of Pol5 with or without K47 mutations, and $0.1 \%(\mathrm{w} / \mathrm{v}) 5$-fluoroorotic acid (5FOA) was used to select against ura $+S$. pombe cells, which is supposed to contain transformed pDblet plasmids.

Mutagenesis and protein tagging. DNA sequences corresponding to Pol5 cDNA were amplified using standard PCR from the $S$. pombe genomic DNA. The primers used are listed in Table II. The resulting PCR product was then cloned into a pCloneNat1 vector [kind gift from Gregan's Lab, Max F. Perutz Laboratories, Vienna Biocenter (VBC), Vienna, Austria]. To create the Pol5 K47N and Pol5 K47R mutant strains, a site directed mutagenesis kit (QuikChange II; Agilent Technologies, Santa Clara, CA, USA) was employed at the corresponding residues using the primers listed in Table II. The resulting plasmids, pCloneNat1pol5 K47N and pCloneNat1-pol5 K47R, were linearized with the restriction enzyme, BstBI, by digesting nucleotides at the start codon, and then transferred into the diploid S. pombe strains. The tetrad analysis of the expected mutation strains was then confirmed by DNA sequencing and used in this study. These wild-type and mutated Pol5 with TAP, HA or FLAG tag were then tagged according to a previously study (23) and online protocol (http://mendel.imp.ac.at/Pombe_tagging/).

Cloning and expression of GST-Esol protein. The full-length Esol cDNA and its different truncated forms, including Rad30 homologue fragment (1-519 or M519*), Rad30 fragment plus the additional second zinc-finger domain (1-568 or R568*) and Ecol homologue fragment (520-871) were amplified from the genomic DNA of $S$. pombe using PCR primers (Table II). The PCR product containing the BamHI and XmaI restriction sites were cloned into the pGEX-4T-1 vector (kind gift from Gerton's Lab, Stowers Institute) in a manner that produces an N-terminally GST tagged Esol protein and its different truncated forms, respectively. The resulting plasmids were transferred into E. coli B121 (DE3) cells (\# 200131; Agilent Technologies), and were amplified and DNA sequence-confirmed. To express the N-terminally GST tagged Esol protein and its different truncated forms, plasmids were transferred into E. coli B121 (DE3) cells and $0.3 \mathrm{mM}$ of IPTG was added to induce the expression of Esol protein in DE3 cells at $25^{\circ} \mathrm{C}$ for $3 \mathrm{~h}$. The glutathione resin was then utilized to pull-down and purify these GST fused proteins, as previously described (24).

TAP purification and liquidchromatography-mass spectrometry (LC-MS/MS) analysis. Trichloroacetic acid (TCA)-precipitated proteins from cultured $S$. pombe strains were resuspended in $30 \mu \mathrm{l}$ of buffer containing $100 \mathrm{mM}$ of Tris- $\mathrm{HCl} \mathrm{pH} 8.5,8 \mathrm{M}$ of urea and $5 \mathrm{mM}$ of Tris(2-carboxylethyl)-phosphine hydrochloride (Pierce, Rockford, IL, USA), and alkylated with $10 \mathrm{mM}$ of iodoacetamide (Sigma, St. Louis, MO, USA). Subsequently, a two-step digestion procedure was applied. In brief, $0.5 \mu \mathrm{g}$ of endoproteinase Lys-C (Roche Applied Science, Indianapolis, IN, USA) was added to each sample and incubated for at least $6 \mathrm{~h}$ at $37^{\circ} \mathrm{C}$. The samples were then diluted with $2 \mathrm{M}$ of urea in $100 \mathrm{mM}$ of Tris- $\mathrm{HCl} \mathrm{pH} 8.5$ and $2 \mathrm{mM}$ of calcium chloride. Subsequently, the samples were digested with $0.5 \mu \mathrm{g}$ of trypsin (Promega, Madison, WI, USA) at $37^{\circ} \mathrm{C}$ overnight with shaking.
The following, the reaction was quenched by the addition of $5 \%$ formic acid, and the peptide mixtures were then loaded onto a $100-\mu \mathrm{m}$ fused silica microcapillary column packed with $8 \mathrm{~cm}$ of reverse-phase material (Aqua; Phenomenex, Torrance, CA, USA) for LC-MS/MS analysis in a Deca-XP ion trap mass spectrometer equipped with a nano-LC electrospray ionization source (Thermo Fisher Scientific, Waltham, MA, USA). The full MS spectra were recorded on the peptides over a 400-1,600 m/z range. Mass spectrometer scan functions and HPLC solvent gradients were controlled by the XCalibur data system (Thermo Fisher Scientific). RAW files were extracted into $m s 2$ file format using RAW Xtract v.1.0. The protein spectra were searched against the Swiss-Prot database using the Mascot software program (http://www.matrixscience.com/).

Immunoprecipitation and western blot analysis. S. pombe cells from a $100-\mathrm{ml}$ culture grown into a 0.8 OD-595 were pelleted and immediately frozen in liquid nitrogen. The $S$. pombe cell pellets were then suspended in $1 \mathrm{ml}$ of lysis buffer containing $50 \mathrm{mM}$ of Tris $\mathrm{pH} 7.5,150 \mathrm{mM}$ of $\mathrm{NaCl}, 0.1 \% \mathrm{NP}-40,1 \mathrm{mM}$ of DTT, $10 \%$ glycerol and a protease inhibitor tablet. The cells were lysed by adding glass beads followed by bead-beating for $60 \mathrm{sec} 5$ times with 1 min intervals on ice. The supernatant was then separated by centrifugation at $14,000 \mathrm{rpm}$ at $4^{\circ} \mathrm{C}$ for $20 \mathrm{~min}$, and subjected to co-immunoprecipitation assay with an anti-FLAG antibody for Eso1-FLAG protein and an anti-HA antibody for Pol5-HA protein, respectively. Specifically, the supernatant was added with $30 \mu \mathrm{l}$ of anti-FLAG or anti-HA affinity gel (\#E6779; EZview ${ }^{\mathrm{TM}}$ Red Anti-HA Affinity Gel; Sigma-Aldrich, Shanghai, China) and incubated at $4^{\circ} \mathrm{C}$ overnight. The following day, the mixtures were washed 5 times with wash buffer containing $50 \mathrm{mM}$ of Tris $\mathrm{pH} 7.5,150 \mathrm{mM}$ of $\mathrm{NaCl}$ and $1 \%$ Triton $\mathrm{X}-100$; they were then eluted in 2X SDS buffer containing $10 \mathrm{mM}$ of Tris $\mathrm{pH} 7.5,1 \mathrm{mM}$ of EDTA and $1 \%$ SDS. The elutes were loaded onto a $4-12 \%$ Bis-Tris gel for sodium dodecyl sulfate-polyacrylamide gel electrophoresis (SDS-PAGE), and the proteins were transferred onto a nitrocellulose membrane (Whatman, Piscataway, NJ, USA). For western blot anaysis, the membrane was blocked with $5 \%$ skimmed milk for $1 \mathrm{~h}$ at room temperature and blotted with primary antibodies [anti-FLAG antibody (1:3,000; \#F3165; Sigma), GST antibody (1:3,000; Abmart, Berkeley Heights, NJ, USA), a-HA antibody (1:10,000; \#sc-7392; Santa Cruz Biotechnology, Santa Cruz, CA, USA)] against HA and FLAG tags (Sigma-Aldrich) respectively, as previously described (24). The glutathione resin bound with variant forms of Esol was incubated with whole cell extracts of $S$. pombe expressing C-terminally HA-tagged Pol5 at $4^{\circ} \mathrm{C}$ overnight, and the resulting samples were subjected to western blot analysis with the anti-HA tag antibody (Sigma-Aldrich).

In vitro acetylation assay. Purified GST-tagged Ecolp and HA affinity gel bound with Pol5-HA was added with a HAT buffer containing $50 \mathrm{mM}$ of Tris $\mathrm{pH} 8.0,5 \%$ glycerol, $0.1 \mathrm{mM}$ of EDTA, $50 \mathrm{mM}$ of $\mathrm{KCl}, 1 \mathrm{mM}$ of DTT, $1 \mathrm{mM}$ of PMSF and $10 \mu \mathrm{M}$ of Acetyl-CoA. This was followed by incubation at $30^{\circ} \mathrm{C}$ for $60 \mathrm{~min}$, as previously described (24). The samples were then subjected to western blot analysis with anti-acetyllysine antibody for acetylation levels and HA antibody as a loading control. 
Table I. Yeast strains used in this study.

\begin{tabular}{llr}
\hline Name & \multicolumn{1}{c}{ Genotype } & Source \\
\hline ZCA002 & h- leu1-32 ura4-D18 his3-D1 ade6-M216 & Our stock \\
ZCA005 & h- leu1-32 ura4-D18 his3-D1 ade6-M216 ESO1-TAP::KAN & Our stock \\
ZCA006 & h- leu1-32 ura4-D18 his3-D1 ade6-M216 ESO1-FLAG::KAN & Our stock \\
ZCB001 & h- leu1-32 ura4-D18 his3-D1 ade6-M216 POL5-HA::NAT & This study \\
ZCB002 & h- leu1-32 ura4-D18 his3-D1 ade6-M216 ESO1-FLAG::KAN POL5-HA::NAT & This study \\
ZCB056 & h- leu1-32 ura4-D18 his3-D1 ade6-M216 POL5-TAP::KAN & This study \\
ZCB057 & h+/h- leu1-32 ura4-D18 his3-D1 ade6-M216/M210 POL5/POL5K47N::NAT & This study \\
ZCB058 & h+/h- leu1-32 ura4-D18 his3-D1 ade6-M216/M210 POL5/POL5K47R::NAT & This study \\
ZCB060 & h? leu1-32 ura4-D18 his3-D1 ade6-M216/M210 POL5K47R::NAT p-pdblet-POL5::URA & This study
\end{tabular}

Table II. Primers used in this study.

\begin{tabular}{ll}
\hline Primers & \multicolumn{1}{c}{ Nucleotide sequences } \\
\hline Wild-type & 5'-atatctcgagttgagaacgttcccatctac-3' \\
Pol5 & 5'-atatggatccatccttgggcttggt-3' \\
Pol5 K47N & 5'-cgttaaaccgtttgaccaatggtctttctagtggtcg-3' \\
mutated & 5'-cgaccactagaaagaccattggtcaaacggtttaacg-3' \\
Pol5 K47R & 5'-ttcgttaaaccgtttgacccgaggtctttctagtggtcgc-3' \\
mutated & 5'-gcgaccactagaaagacctcgggtcaaacggtttaacgaa-3' \\
Pol5 & 5'-tacaacctcgattgtgag-3' \\
mutated & \\
\hline
\end{tabular}

Pol5, polymerase 5 .

\section{Results}

Esol protein interaction with Pol5 protein in S. pombe. To identify proteins that can physically interact with Eso1 protein, Eso1 cDNA was tagged with a TAP tag at the $\mathrm{C}$-terminus and this fusion protein was expressed under its endogenous promoter (25) using a protocol we have previously described (25). The proteins binding to Eso1-TAP protein were then purified using immunoprecipitation and mass spectrometry followed by TAP. Our data revealed that Eso1 protein was able to bind to $\mathrm{Cdc} 2$, Pol5 and Cdc21, as well as other proteins (Fig. 1A). The following experiments focused on Pol5 protein due to its function in rRNA synthesis and cell proliferation. The specificity of their binding was confirmed by immunoprecipitating the whole cell extracts of $S$. pombe from the wild-type strain, the strain that only expressed C-terminally FLAG-tagged Eso1, and the strain that expressed both C-terminally HA-tagged Pol5 and FLAG-tagged Eso1 with anti-HA or FLAG antibodies. Following western blot analysis using anti-FLAG or $\alpha$-HA antibody, their binding and interaction (i.e., the proper expression of Eso1-FLAG and Pol5-HA) were directly confirmed from the indicated strains (Fig. 1B). Specifically, Pol5-HA protein could only be detected from whole cell extracts of strains that express both HA-tagged Pol5 and FLAG-tagged Esol, indicating the specificity of their interaction.
However, given the characteristics of the fusion Eso1 protein in $S$. pombe, which expresses both budding yeast Ecol and Rad30 homologue (26), our present data was unable to distinguish the binding of Pol5 protein to Eco1 protein from the binding of Pol5 to the Rad30 homologue of Eso1 protein. Thus, we amplified DNA sequences of Rad30 homologue fragment (1-568 or R568*), Rad30 fragment plus the additional second zinc-finger domain (1-519 or M519*), Eco1 homologue fragment (520-871) and the full length Esol cDNA using genomic DNA (Fig. 1C). We then inserted these amplicants into pGEX-4T-1 to produce the expected form of Esol protein with the N-terminal GST-tag (Fig. 1D). Following GST pull-down assays and western blot analysis with anti-GST antibody, the correct expression of Eso1 proteins were confirmed (Fig. 1D). These proteins were then utilized to pull-down Pol5 in the whole cell lysis of S. pombe expressing C-terminally HA-tagged Pol5 for western blot analysis with anti- $\alpha-\mathrm{HA}$ antibody. We found that the results were consistent with our first set of data, which showed that Pol5 protein binds to Eso1 protein from either Eso1-FLAG and Pol5-HA co-immunoprecipitation or mass spectrometry analysis following the purification of Esol by TAP (Fig. 1); Pol5-HA was able to pull-down the full-length recombinant GST-Esol protein. Strikingly, Pol5-HA could have also pulled-down the Eco1 homology part of Eso1, but not the Rad30 part or Rad30 part plus additional zinc finger domain of Ecol protein (Fig. 1E), suggesting that the Ecol homology part of Esol mediated binding to the Pol5 protein.

Mass spectrometry identification of Pol5 protein acetylation sites. C-terminal TAP tagging and Pol5 protein expression in vitro was established according to a protocol described in a previous study (23). Proteins were purified using an anti-TAP tag antibody and separated using SDS-PAGE. A prominent protein band of approximately $120 \mathrm{kDa}$ was found after silver staining (Fig. 2A), which was in agreement with the predicted molecular weight of Pol5 protein plus TAP tag. Possible post-translational modifications of this purified Pol5 protein were then analyzed using mass spectrometry, and several acetylation or trimethylation modification sites in the lysine residues of Pol5 protein were identified (Fig. 2B). The Pol5 K47 residue attracted our attention, because there was a $100 \%$ modification ratio (although the number of the total spectrum count was low); the DNA sequence alignment of S.pombe 
A

\begin{tabular}{cccc}
\hline Name & $\begin{array}{c}\text { Peptide } \\
\text { Count }\end{array}$ & $\begin{array}{c}\text { Distributed } \\
\text { Spectral Count }\end{array}$ & $\begin{array}{c}\text { Sequence } \\
\text { Coverage (\%) }\end{array}$ \\
\hline Eso1 & 56 & 1119 & 28.2 \\
Cdc2 & 2 & 2 & 11.78 \\
Pol5 & 2 & 3 & 3.55 \\
Cdc21 & 2 & 3 & 3.07 \\
Cdc15 & 1 & 2 & 1.4 \\
Rad25 & 4 & 10 & 20 \\
Rad24 & 4 & 5 & 20.74 \\
\hline
\end{tabular}

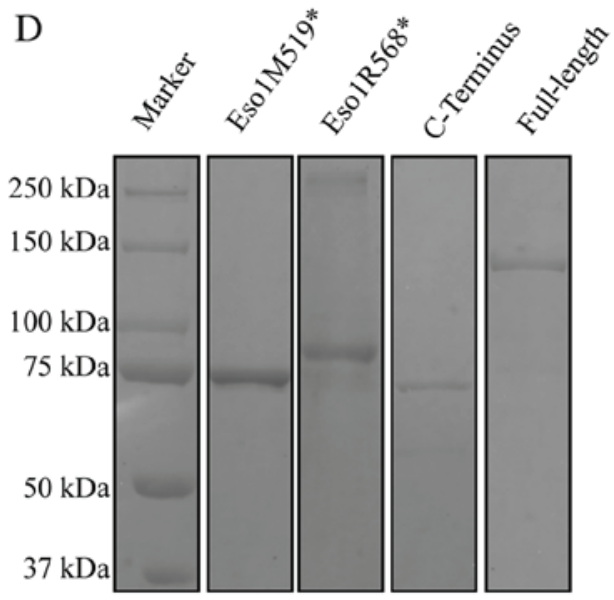

$\mathrm{E}$

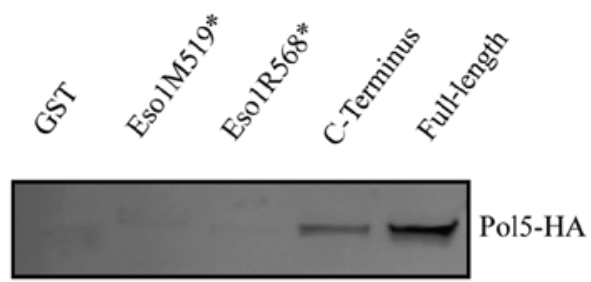

IP: Pol5-HA

$\mathrm{C}$

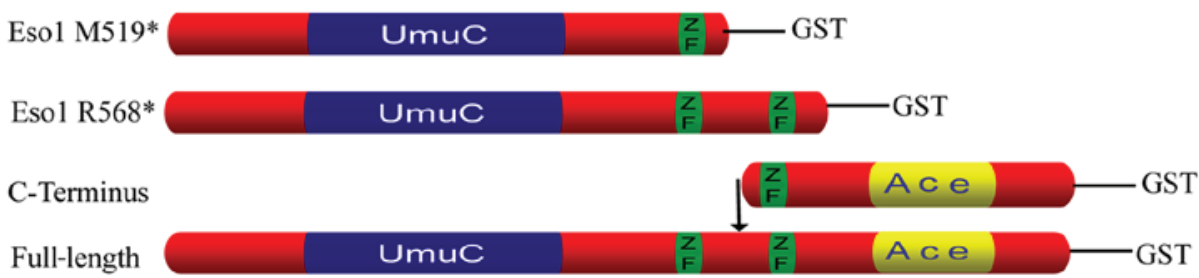

Figure 1. Eso1 interaction with Pol5 in Schizosaccharomyces pombe (S. pombe). (A) Immunoprecipitation and mass spectrometry identification of Eso1 binding partners in S. pombe. (B) Immunoprecipitation-western blot analysis: $50 \mu \mathrm{g}$ of whole-cell lysis from $S$. pombe expressing C-terminally FLAG-tagged Esol and HA-tagged Pol5 were immunoprecipitated using an anti-HA antibody, and subjected with western blot analysis with an anti-FLAG antibody. (C) Illustration of different forms of GST-fusion $S$. pombe Esol protein purified from E. coli. Different truncated forms of GST-fusion Esol protein were constructed and expressed as indicated. The * sybmol indicates the induced stop codon at Eso1 cDNA to construct a truncated Eso1 protein (1-568 and 1-519); the arrow indicates the position of the start site of the C-terminal truncation (520-871) in Esol cDNA. (D) Coomassie staining of SDS-PAGE gel. GST-Esol and its different truncated forms were expressed and detected by SDS-PAGE and Coomassie staining. (E) Immunoprecipitation. Whole-cell lysis extracted from S. pombe expressing C-terminally HA-tagged Pol5 was immunoprecipitated using GST beads bound to GST-fused Esol protein or different truncated forms of GST-fused Eso1 protein, as indicated. Data indicated that only the Eso1-GST and C-terminus Esol protein were able to pull down the Pol5-HA protein.

and $S$. cerevisiae indicate that $\mathrm{K} 47$ residue is well conserved in budding yeast (Fig. 2C).

Acetylation of Pol5 protein $K 47$ residue mediated S. pombe viability. The effects of Pol5 K47 acetylation on $S$. pombe cells were assessed, and pol5-K47R/pol $5^{+}$heterozygous diploids were prepared. Following sporulation, only two spores in each diploid grew (Fig. 3A). DNA sequencing analysis was then performed. The results revealed that all viable spores contained a wild-type (non-mutated) allele of the Pol5 gene, indicating that $S$. pombe expressing mutant Pol5 K47R was unable to survive. Moreover, since arginine $(\mathrm{R})$ is structurally similar to lysine $(\mathrm{K})$, which does not undergo acetylation change, we found that arginine substitution of lysine led to the absence of the acetylation of Pol5 K47; this resulted in the lethality to
S. pombe. In addition, our data also revealed that Pol5 K47N acetyl-mimetic mutant $S$. pombe cells grew normally (Fig. 3A).

Furthermore, we constructed the shuffle plasmid by integrating the Pol5 coding region cDNA plus the 200-bp upstream and downstream sequences into the pDblet vector. The transformation of this plasmid into $S$. pombe fully rescued the growth of Pol5 K47R mutants (Fig. 3). Subsequently, 5FOA was added into $S$. pombe to select against the expression of Ura4+ in pDblet-Pol5 plasmid-transferred S. pombe. Consistent with the data on Pol5 K47R mutation without pDblet-Pol5 plasmid, our data revealed that $S$. pombe growth was limited and lethal (Fig. 3B).

Esol acetylation of Pol5 protein $K 47$ residue mediates $S$. pombe viability. We explored whether Esol protein is able 
A

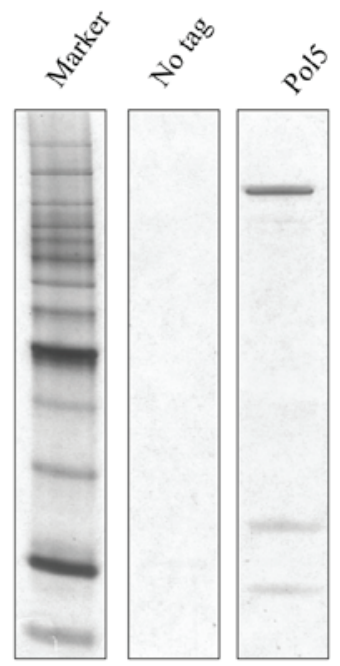

$\mathrm{C}$

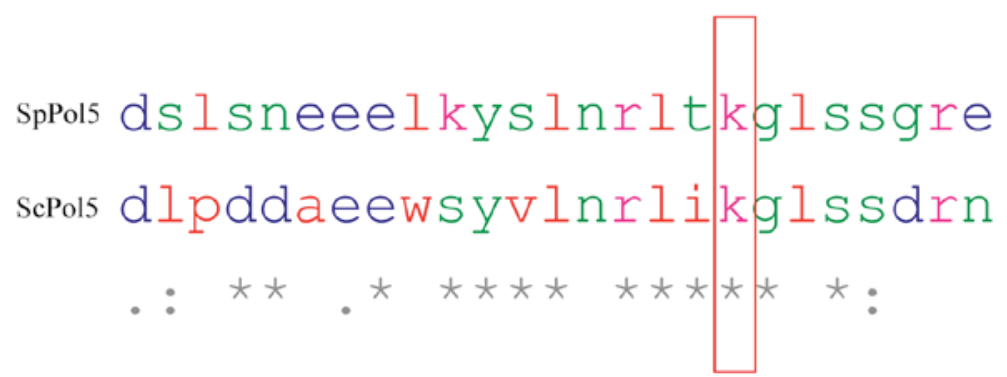

B

\begin{tabular}{llrrr}
\hline \multicolumn{1}{r}{$\begin{array}{r}\text { Residue } \\
\text { Position }\end{array}$} & PTM-type & $\begin{array}{r}\text { Total Spectrum } \\
\text { Count }\end{array}$ & $\begin{array}{c}\text { Modified } \\
\text { Spectrum Count }\end{array}$ & Ratio: Modified/total \\
\hline K39 & Acetylation/Trimethylation & 521 & 12 & 2.3 \\
K47 & Acetylation/Trimethylation & 8 & 8 & 100 \\
K220 & Acetylation/Trimethylation & 111 & 1 & 0.9 \\
K542 & Acetylation/Trimethylation & 850 & 37 & 4.35 \\
K792 & Acetylation/Trimethylation & 110 & 3 & 2.73 \\
K823 & Acetylation/Trimethylation & 169 & 2 & 1.18 \\
K886 & Acetylation/Trimethylation & 71 & 3 & 4.23 \\
\hline
\end{tabular}

Figure 2. Tandem affinity purification (TAP) and characterization of Pol5 protein from Schizosaccharomyces pombe (S. pombe). (A) TAP-tagged Pol5 cDNA was transferred into $S$. pombe, and whole cell lysis was extracted from $S$. pombe. Pol5 protein was then purified by the TAP system. Purified proteins were separated by SDS-PAGE and visualized by silver staining. (B) Illustration of potential post-translational modification sites in Pol5 protein. (C) Mass spectrometry identification of the Po15 K47 site. The acetylated lysine residue of Pol5 protein in S. pombe is conserved to K53 of Pol5 in budding yeast (a red box). In the alignment, asterisks indicate identity, while the colons and dots indicate similarity between amino acids.

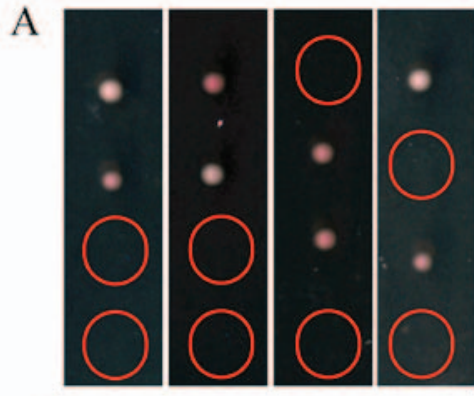

Pol5-K47R (Non-acetylated)
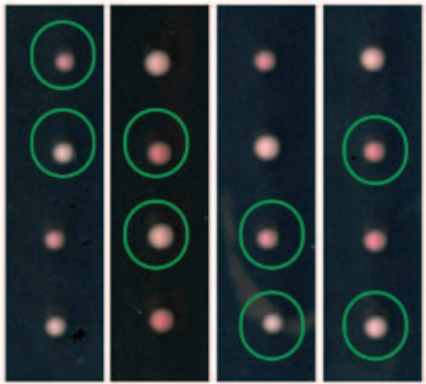

Pol5-K47R (Acetyl mimic)
B

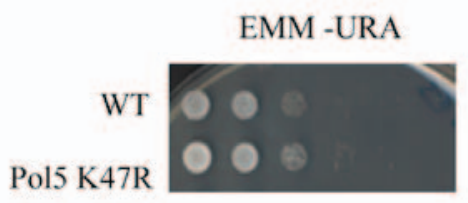

C

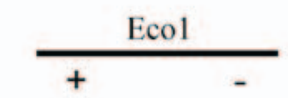

FOA
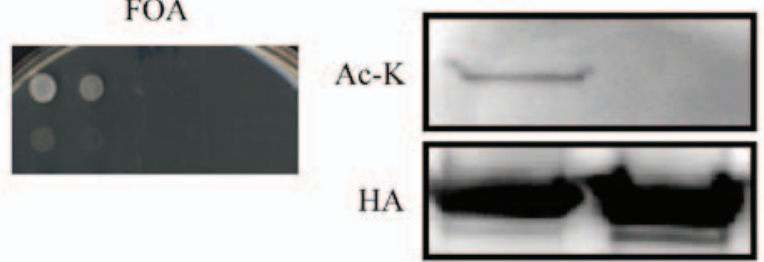

Figure 3. Lethality of non-acetylated K47 residue mutation in Pol5 protein in Schizosaccharomyces pombe (S.pombe).(A) POL5K47R/POL5 and POL5 K47N/POL5 heterozygous diploids were sporulated, and the viability of each tetrad was analyzed. POL5 K47R mutant spores are labeled with red circles, and POL5 K47N mutant spores are labeled with green circles. (B) Wild-type (WT) and Pol5 K47R cells containing pDblet-pol5 plasmids were plated on EMM-URA and YES 5'FOA plates. (C) Pol5 was acetylated by Ecol in vitro. Pol5-HA was immunoprecipitated on an HA affinity gel from whole cell extracts of S. pombe cells expressing C-terminally HA tagged Pol5. The beads were then incubated with or without Ecol in HAT buffer present with acetyl coenzyme A. 
to acetylate Pol5 using acetylation assay following a protocol described in a previous study (27). We found that Pol5-HA protein was immunoprecipitated with EZview ${ }^{\mathrm{TM}}$ Red Anti-HA Affinity Gel from $S$. pombe. This was then incubated with the recombinant Ecol protein purified from E. coli via the GST tag. Pol5 protein acetylation was detected by western blot analysis with an anti-acetyl-lysine antibody following the addition of Ecol protein (Fig. 3C).

\section{Discussion}

In the present study, we first performed immunoprecipitation and mass spectrometry assays to identify several candidate proteins that bind to Eso1 protein (i.e., Cdc2, Cdc21, Cdc15, $\operatorname{Rad} 24, \operatorname{Rad} 25$ and Pol5). Functionally, Cdc2, as a serine/threonine kinase, is a highly conserved protein and a key player in the regulation of cell cycle progression $(22,28)$. Cdc21 is a member of the MCM family of nuclear proteins, which can regulate DNA replication and cell cycle progression (29). $\mathrm{Cdc} 15$ is also involved in the formation of the cytokinetic contractile ring (28). Moreover, $\operatorname{Rad} 24$ and $\operatorname{Rad} 25$ are c ell cycle checkpoint proteins that belong to the highly conserved 14-3-3 protein family (29-31), which regulates cell cycle arrest and DNA damage repair in response to UV light-induced DNA damage (24-27). Pol5 protein functions in yeast rDNA transcription $(17,18)$ and the human homologue of Pol5, Mybbpla, demonstrating functions in rDNA transcription, early embryonic development and tumorigenesis $(19,20)$. Thus, given the important functions of Esol protein in cells, including the sister chromatin cohesion complex and mediation of DNA repair (2), it seems reasonable to hypothesize that the binding of Esol to these proteins illustrates the functions of Esol protein.

Furthermore, we focused on the interaction of Pol5 protein with Esol protein to assess their regulation of $S$. pombe viability. We performed a co-immunoprecipitation assay to further confirm the binding of Ecol to Pol5 protein and found that Pol5 protein only bound to Esol protein, but not to the Rad30 part or Rad30 part, plus the additional zinc finger domain of Ecol protein, indicating that their binding was specific. Upon their binding, Eso1 protein was able to acetylate Pol5 protein. Our confirmative evidence indicated that there were several acetylation sites in Pol5 protein lysine residues (Fig. 2B) and that arginine substitution of lysine led to the lack of Pol5 K47 acetylation, which resulted in the lethality of $S$. pombe; whereas for Pol5 K47N, the acetyl-mimetic mutant had normal $S$. pombe proliferation; thus, the Esol acetylation site in Pol5 protein could be at the Pol5 K47. However, at present, there are no Pol5 K47 acetylation antibodies available; thus, a definitive proof would require the involvement of a mass-spec analysis of the acetylation on Pol5 before and after incubation with Esol protein. Future studies are required to further verify the Esol acetylating Pol5 K47 site, although our present data suggest that Pol5 protein acetylation was essential in the regulation of $S$. pombe viability.

In conclusion, previous studies have revealed the essential role of Eco1/Esol protein in the formation of the cohesion complex between the two sister chromatids, and that the acetylmimetic mutation, Smc3 K47N, made Ecol protein dispensable. In the present study, we found that the $S$. pombe carrying nonacetylatable mutations of Pol5 protein (i.e., Pol5 K47R) was unable to survive, indicating the essential role of Pol5 acetylation in S. pombe. However, this study was not able to rule out whether other acetyltransferases, apart from Eso1, acetylate Pol5 protein. Future studies are warranted to verify Eso1, particularly the acetylate Pol5 K47 site; although the present study suggested so.

\section{Acknowledgements}

The authors would like to thank Dr Juraj Gregan of Max F. Perutz Laboratories, Department of Chromosome Biology, University of Vienna, Vienna, Austria; and the Stowers Proteomics and Molecular Biology groups, MO, USA. This study was supported by a grant from the National Natural Science Foundation of China (\#81672122), and Jilin Provincial Science and Technology Agency (\#20160414048GH).

\section{References}

1. Skibbens RV, Corson LB, Koshland D and Hieter P: Ctf7p is essential for sister chromatid cohesion and links mitotic chromosome structure to the DNA replication machinery. Genes Dev 13: 307-319, 1999.

2. Tanaka K, Yonekawa T, Kawasaki Y, Kai M, Furuya K, Iwasaki M, Murakami H, Yanagida M and Okayama H: Fission yeast Esolp is required for establishing sister chromatid cohesion during S phase. Mol Cell Biol 20: 3459-3469, 2000.

3. Unal E, Heidinger-Pauli JM and Koshland D: DNA doublestrand breaks trigger genome-wide sister-chromatid cohesion through Ecol (Ctf7). Science 317: 245-248, 2007.

4. Nasmyth K and Schleiffer A: From a single double helix to paired double helices and back. Philos Trans R Soc Lond B Biol Sci 359: 99-108, 2004

5. Hirano T: Chromosome cohesion, condensation, and separation. Annu Rev Biochem 69: 115-144, 2000.

6. Nasmyth K: Disseminating the genome: Joining, resolving, and separating sister chromatids during mitosis and meiosis. Annu Rev Genet 35: 673-745, 2001.

7. Koshland DE and Guacci V: Sister chromatid cohesion: The beginning of a long and beautiful relationship. Curr Opin Cell Biol 12: 297-301, 2000.

8. Zhang J, Shi X, Li Y, Kim BJ, Jia J, Huang Z, Yang T, Fu X, Jung SY, Wang Y, et al: Acetylation of Smc3 by Eco1 is required for S phase sister chromatid cohesion in both human and yeast. Mol Cell 31: 143-151, 2008.

9. Unal E, Heidinger-Pauli JM, Kim W, Guacci V, Onn I, Gygi SP and Koshland DE: A molecular determinant for the establishment of sister chromatid cohesion. Science 321: 566-569, 2008.

10. Rolef Ben-Shahar T, Heeger S, Lehane C, East P, Flynn H, Skehel $\mathrm{M}$ and Uhlmann F: Ecol-dependent cohesin acetylation during establishment of sister chromatid cohesion. Science 321: 563-566, 2008.

11. Feytout A, Vaur S, Genier S, Vazquez S and Javerzat JP: Psm3 acetylation on conserved lysine residues is dispensable for viability in fission yeast but contributes to Eso1-mediated sister chromatid cohesion by antagonizing Wpl1. Mol Cell Biol 31: 1771-1786, 2011.

12. Vega H, Waisfisz Q, Gordillo M, Sakai N, Yanagihara I, Yamada M, van Gosliga D, Kayserili $\mathrm{H}, \mathrm{Xu} \mathrm{C}$, Ozono K, et al: Roberts syndrome is caused by mutations in ESCO2, a human homolog of yeast ECO1 that is essential for the establishment of sister chromatid cohesion. Nat Genet 37: 468-470, 2005.

13. Dorsett D: Cohesin: Genomic insights into controlling gene transcription and development. Curr Opin Genet Dev 21: 199-206, 2011.

14. Chen Z, McCrosky S, Guo W, Li H and Gerton JL: A genetic screen to discover pathways affecting cohesin function in Schizosaccharomyces pombe identifies chromatin effectors. G3 (Bethesda) 2: 1161-1168, 2012.

15. Bose T, Lee KK, Lu S, Xu B, Harris B, Slaughter B, Unruh J, Garrett A, McDowell W, Box A, et al: Cohesin proteins promote ribosomal RNA production and protein translation in yeast and human cells. PLoS Genet 8: e1002749, 2012.

16. Xu B, Lee KK, Zhang L and Gerton JL: Stimulation of mTORC1 with L-leucine rescues defects associated with Roberts syndrome. PLoS Genet 9: e1003857, 2013.

17. Yang W, Rogozin IB and Koonin EV: Yeast POL5 is an evolutionarily conserved regulator of rDNA transcription unrelated to any known DNA polymerases. Cell Cycle 2: 120-122, 2003. 
18. Shimizu K, Kawasaki Y, Hiraga S, Tawaramoto M, Nakashima N and Sugino A: The fifth essential DNA polymerase phi in Saccharomyces cerevisiae is localized to the nucleolus and plays an important role in synthesis of rRNA. Proc Natl Acad Sci USA 99: 9133-9138, 2002.

19. Mori S, Bernardi R, Laurent A, Resnati M, Crippa A, Gabrieli A, Keough R, Gonda TJ and Blasi F: Myb-binding protein $1 \mathrm{~A}$ (MYBBP1A) is essential for early embryonic development, controls cell cycle and mitosis, and acts as a tumor suppressor. PLoS One 7: e39723, 2012.

20. Hochstatter J, Hölzel M, Rohrmoser M, Schermelleh L, Leonhardt H, Keough R, Gonda TJ, Imhof A, Eick D, Längst G, et al: Myb-binding protein 1a (Mybbpla) regulates levels and processing of pre-ribosomal RNA. J Biol Chem 287: 24365-24377, 2012.

21. Sabatinos SA and Forsburg SL: Molecular genetics of Schizosaccharomyces pombe. Methods Enzymol 470: 759-795, 2010.

22. Gregan J, Rabitsch PK, Rumpf C, Novatchkova M, Schleiffer A and Nasmyth K: High-throughput knockout screen in fission yeast. Nat Protoc 1: 2457-2464, 2006.

23. Cipak L, Spirek M, Novatchkova M, Chen Z, Rumpf C, Lugmayr W, Mechtler K, Ammerer G, Csaszar E and Gregan J: An improved strategy for tandem affinity purification-tagging of Schizosaccharomyces pombe genes. Proteomics 9: 4825-4828, 2009.

24. Xiong B, Lu S and Gerton JL: Hos1 is a lysine deacetylase for the Smc3 subunit of cohesin. Curr Biol 20: 1660-1665, 2010.
25. Chen Z, Cao H, Guo W and Lu Y: Identification of two forms of the Esol protein in Schizosaccharomyces pombe. Cell Biol Int 38: 682-688, 2014.

26. Madril AC, Johnson RE, Washington MT, Prakash L and Prakash S: Fidelity and damage bypass ability of Schizosaccharomyces pombe Esol protein, comprised of DNA polymerase eta and sister chromatid cohesion protein Ctf7. J Biol Chem 276: 42857-42862, 2001.

27. Lu S, Goering M, Gard S, Xiong B, McNairn AJ, Jaspersen SL and Gerton JL: Ecol is important for DNA damage repair in S. cerevisiae. Cell Cycle 9: 3315-3327, 2010.

28. Fankhauser C, Reymond A, Cerutti L, Utzig S, Hofmann K and Simanis V: The $S$. pombe cdc15 gene is a key element in the reorganization of F-actin at mitosis. Cell 82: 435-444, 1995.

29. Ford JC, al-Khodairy F, Fotou E, Sheldrick KS, Griffiths DJ and Carr AM: 14-3-3 protein homologs required for the DNA damage checkpoint in fission yeast. Science 265: 533-535, 1994.

30. Ozoe F, Kurokawa R, Kobayashi Y, Jeong HT, Tanaka K, Sen K, Nakagawa T, Matsuda $\mathrm{H}$ and Kawamukai M: The 14-3-3 proteins Rad24 and Rad25 negatively regulate Byr2 by affecting its localization in Schizosaccharomyces pombe. Mol Cell Biol 22: 7105-7119, 2002

31. van Heusden GP and Steensma HY: Yeast 14-3-3 proteins. Yeast 23: 159-171, 2006. 